\title{
Los trabajadores de empacadoras de yuca en comunidades de La Fortuna, San Carlos, y Sector Ángeles, San Ramón, Costa Rica. Una aproximación sociológica
}

Francisco Rodríguez Barrientos '

Óscar Córdoba Artavia ${ }^{2}$

Fecha de recepción: 25 de abril del 2011

Fecha de aceptación: 17 de octubre del 2011

Rodríguez, F; Córdoba, O. Los trabajadores

de empacadoras de yuca en comunidades de La Fortuna, San Carlos, y Sector Ángeles, San Ramón, Costa Rica. Una aproximación sociológica. Tecnología en Marcha. Vol. 25, N². Abril-Junio 2012 Pág 50-60.

I Sociólogo. Escuela de Ciencias y Letras, Tecnológico de Costa Rica, sede San Carlos. Teléfono: 2401-3065. Correo electrónico: franroba@gmail.com

2 Ingeniero en Producción. Escuela de Administración de Empresas, Tecnológico de Costa Rica, sede San Carlos. Correo electrónico: ocartavia@gmail.com 


\section{Resumen}

El presente artículo se deriva del proyecto de investigación Mejora en la eficiencia de la cadena productiva de la yuca en el distrito de La Fortuna, San Carlos, Costa Rica. El objetivo fundamental del proyecto consistía en mejorar la eficiencia y la productividad en las diversas etapas del proceso agro productivo de la yuca. La investigación se realizó en una franja territorial que abarcó un sector del distrito de La Fortuna, cantón de San Carlos, provincia de Alajuela, Costa Rica, un importante lugar de producción yuquera, y una porción del distrito de Peñas Blancas (cantón San Ramón, provincia de Alajuela). La franja territorial seleccionada se halla dentro de la Región Huetar Norte costarricense, cuyo sector ligado a la producción, procesamiento y comercialización de yuca constituyó la población meta de la investigación. El proyecto tuvo una duración de tres años (20062008).

El presente artículo presenta un bosquejo comparativo entre trabajadores costarricenses y migrantes nicaragüenses que laboran en procesadoras de yuca de la franja territorial estudiada respecto a sus condiciones económicas, sociales y laborales. El artículo enfatiza algunas variables fundamentales como los niveles de ingreso y pobreza, los niveles de escolaridad y la tenencia de seguro social. La información ofrecida es una simple aproximación a una realidad compleja y cambiante, cuyos resultados se busca puedan servir de guía o de hipótesis de trabajo para ulteriores estudios que aborden esta problemática de forma más amplia y sistemática.

\section{Palabras clave}

La Fortuna de San Carlos, trabajadores de empacadoras, trabajadores costarricenses, migrantes nicaragüenses, niveles de pobreza, grado de escolaridad, tenencia de seguro social.

\section{Abstract}

This article derives from the research project Improvement in the efficiency of the productive chain of cassava in the district of Fortune, San Carlos, Costa Rica. The main objective of the project consisted on improving the efficiency and productivity in the various stages of the agro productive process of the cassava. The research was conducted in a strip of land covering an area of the district of La Fortuna, canton of San Carlos, Alajuela, Costa Rica, traditionally a major cassava growing production site, and an area of the district of Peñas Blancas (canton of San Ramón, Alajuela province). The strip of land selected is within the North Huetar Costa Rica. This population, connected to the production sector, processing and marketing of cassava, was the goal of the research population. The project lasted three years (2006-2008).

This article presents a comparative sketch between Costa Rican and Nicaraguan migrant workers working in cassava processing territorial plants studied in regards to their economic, social and labor conditions. The article focuses on some key variables such as income and poverty levels, educational levels and social security. The information provided is a simple approach to a complex and changing reality, and whose results can guide search or working hypothesis for further studies to address this issue more widely and systematically.

Key words

La Fortuna, San Carlos, plant workers, Costa Rican workers, Nicaraguan migrant, poverty levels, education levels, social security. 


\section{Justificación}

La franja seleccionada para el presente estudio se ubica mayormente en el distrito de La Fortuna, cantón de San Carlos y en una pequeña fracción perteneciente al distrito de Peñas Blancas, cantón de San Ramón. El distrito de La Fortuna es una de las principales zonas yuqueras de Costa Rica y concentra gran cantidad de fincas dedicadas a dicha actividad y varias empacadoras (catorce solo en la franja). Esta franja es un ejemplo de integración relativamente exitoso a la nueva agroexportación alentada por sucesivos gobiernos costarricenses desde inicios de los años 1980. Por estas razones se escogió dicha franja para un estudio sobre los distintos factores que pueden estar incidiendo en la eficiencia de la cadena productiva de la yuca y la situación socioeconómica y laboral ligada a dicha cadena.

La pujanza de los productos no tradicionales de exportación en San Carlos atrajeron a la zona gran cantidad de trabajadores nicaragüenses que constituyen un elevado porcentaje de la fuerza laboral en las empacadoras de yuca y otros productos agrícolas de exportación ubicadas tanto en la franja estudiada, los datos del presente estudio así lo corroboran, como en el resto del territorio sancarleño. Para la investigación era importante comparar la situación laboral y socioeconómica de los trabajadores de las empacadoras de yuca de la franja según nacionalidad. Los resultados obtenidos y su respectiva discusión se ofrecen en el presente artículo.

\section{Breve caracterización de la franja territorial investigada}

De los escasos registros disponibles actualmente, se conoce que la zona en estudio se empezó a colonizar a fines de la década de 1930 (Vargas, 1986). Un hecho importante fue el establecimiento de varios asentamientos campesinos en los años 1970, con la participación del Instituto de Desarrollo Agrario (IDA), como Zona Fluca, Sector Ángeles, El Burrito y El Brujo, los cuales en los primeros años se dedicaban a la ganadería, al cultivo de plátano y a los granos básicos, pero desde 1990 incursionan en la producción de raíces y tubérculos, con el predominio de la yuca, alentados por las empacadoras que empiezan a instalarse en los alrededores (Rodríguez, 2003 a y b).
Durante el periodo 1950-1990 la principal actividad económica fue la ganadería de carne en los inicios y de doble propósito a partir de 1970. La mayor parte de los suelos fueron transformados en pastizales luego de una deforestación masiva, que en el periodo citado, también afectó a gran parte del territorio sancarleño (Rodríguez, 2006). Sin embargo, la nueva estrategia exportadora iniciada a principios de los años ochenta trajo un importante cambio en el uso del suelo porque los pastizales fueron cediendo terreno frente a nuevos cultivos como los tubérculos, el arroz, el plátano y la caña de azúcar (Estado de la Nación, 1997). A pesar del relativo declive del sector pecuario, los repastos siguen ocupando buena parte de los suelos productivos, situación que confirman estudios más recientes (Rodríguez, 2003 a, 2006).

En la franja estudiada los agricultores debieron asumir el reto de lo que en su momento se llamó la "reconversión productiva" (Rivera y Román, 1990), pues la producción de alimentos era una de sus actividades más relevantes; quienes no pudieron hacerlo, debieron abandonar la actividad, vender sus parcelas, emplearse en las empacadoras que empiezan a proliferar en la franja desde mediados de los años ochenta o emigrar a los centros urbanos del Valle Central (Rodríguez, 2006).

Los datos muestran -y la presente investigación lo confirma -que, en la franja en estudio, muchos agricultores pudieron integrase bien a los grandes mercados internacionales, lo que ha repercutido en un mejoramiento del nivel de vida de parte de su población; sin embargo, la yuca es fundamentalmente un producto étnico (latinoamericano, asiático y africano) en los principales mercados consumidores (Estados Unidos y Europa). Lo anterior significa que el crecimiento de la exportación de yuca hacia los principales mercados consumidores depende en buena medida del progreso económico y social que obtengan las poblaciones de origen latinoamericano y africano.

Esta circunstancia hace vulnerable a las exportaciones de yuca cuando esos grupos étnicos se ven afectados por crisis económicas en los países donde residen, como la que afecta a Estados Unidos y España desde el 2007 y que ha dejado sin empleo a decenas de miles de inmigrantes latinoamericanos. 
La constante oscilación de los precios de la yuca en el mercado internacional es otra vulnerabilidad que deben enfrentar los productores de yuca de la franja investigada y que repercute sobre las unidades campesinas que la habitan.

Como en otros lugares de Costa Rica, para no hablar ya de San Carlos, la franja estudiada ha visto la presencia de gran cantidad de nicaragüenses que se han empleado en las fincas agrícolas y en las empacadoras. Estos inmigrantes juegan un papel de gran importancia en el mercado laboral de la franja y a futuro, sobre todo en la medida que decidan asentarse definitivamente en su nuevo medio, tendrán un peso cada vez mayor en la vida económica, social y cultural del país. Es obvio que la Sociología Rural tiene aquí una rica veta de estudio.

\section{Materiales y métodos}

La investigación se realizó en el distrito de La Fortuna y una porción del distrito de Peñas Blancas (Cantón San Ramón), en la Región Huetar Norte, cuyo sector ligado a la producción, procesamiento y comercialización de yuca es la población meta.

\section{Elección de la muestra}

En la franja seleccionada para investigación se encontraron un total de 14 empacadoras. Aunque la investigación originalmente se enfocó en las fincas agrícolas y en la parte de proceso de las empacadoras, el equipo investigador decidió hacer un estudio exploratorio sobre la situación de los trabajadores en las empacadoras, tanto costarricenses como nicaragüenses. De esta manera se encuestaron a 50 trabajadores de las cinco empacadoras más grandes ubicadas en la franja: 28 nicaragüenses y 22 costarricenses. El propósito fue recabar información cuyos resultados puedan servir de base para investigaciones futuras más detalladas y precisas y que trabajen con muestras más amplias y representativas.

\section{Técnicas de investigación utilizadas}

En lo que se refiere a la parte de proceso, se decidió encuestar todas las empacadoras de yuca presentes en la franja en estudio; en total fueron 14. Los resultados ofrecidos en los gráficos 1, 2 y 3 se obtuvieron de la información proporcionada por los dueños o gerentes de las empacadoras para todos sus trabajadores.
Para recolectar la información de los trabajadores de las plantas procesadoras se confeccionaron dos cuestionarios: uno para nicaragüenses y otro para costarricenses. Se hicieron dos pruebas por cada instrumento con el propósito de validarlo. Una vez realizados los ajustes necesarios se procedió a aplicar el instrumento a los trabajadores de las cinco empacadoras seleccionadas. Los datos presentados en los gráficos 4 y 5, así como la información correspondiente al seguro social se obtuvieron de estas encuestas.

La información recolectada fue registrada y procesada en hojas especialmente diseñadas en el programa Windows Excel 2003.

\section{Resultados y discusión}

En el gráfico I se presenta la distribución de la mano de obra en las empacadoras por tipo de actividad. Como era de esperar, la mayoría de los trabajadores (83\%) son de proceso, siendo pocos quienes se desempeñan en las tareas administrativas. En cuanto a la distribución por género, hay un claro predominio de las mujeres, quienes representan el 63\% de la fuerza laboral en las plantas procesadoras (gráfico 2). Este dato confirma los resultados de otras investigaciones realizadas dentro y fuera de la zona de San Carlos, que muestran la gran presencia femenina en las plantas transformadoras de productos no tradicionales de exportación, por ejemplo Weller (1996), Stamm (1996) y Rodríguez et al. (2004). En un trabajo hecho en el ámbito laboral de San Carlos entre los años 2002-2003, Romero et al. (2003) hallaron, asimismo, una alta presencia de mujeres en las empacadoras del cantón, especialmente en las de piña y en las de raíces y tubérculos.

Esta característica coincide con el estudio de Cordero y Mora (1998) sobre el mercado laboral costarricense, en el cual hallaron que en el sector agrícola que los autores denominaron "modernizado", y que en lo fundamental abarcaba a los productos no tradicionales de exportación, la presencia femenina era claramente superior a la masculina, especialmente en las empacadoras. Algunos de los estudios citados, como los de Weller (1996) y Cordero y Mora (1998), coinciden en señalar, no obstante, que la incorporación de las mujeres en las labores agrícolas es menos significativa. 


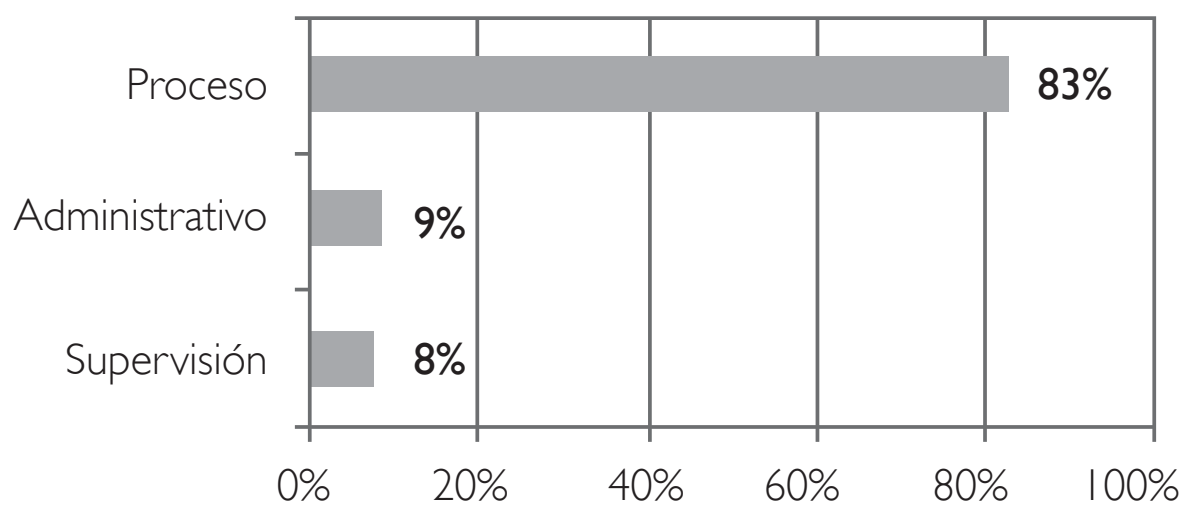

Gráfico I. Distribución de la mano de obra en las empacadoras de yuca por tipo de actividad (porcentaje).

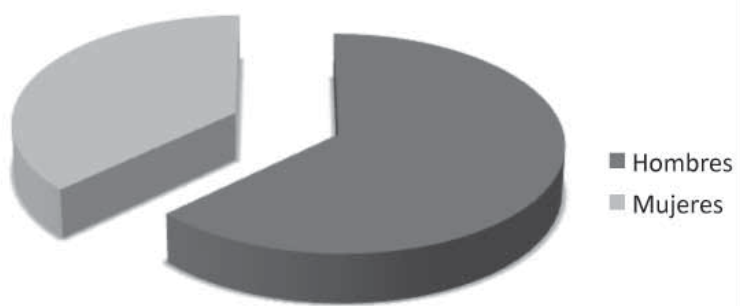

Gráfico 2. Distribución de la mano de obra en las empacadoras de yuca por género (porcentaje).

Para autores como Cordero y Mora (1998) y Morales y Castro (2008), la alta presencia de mujeres en las empacadoras obedece, entre otros factores, a los menores salarios que se les paga; por eso a las empresas les resulta rentable su contratación. Morales y Castro (2008) mencionan que en empacadoras sancarleñas en abril del 2002 se pagaba 335 colones/hora a los hombres, pero en cambio las mujeres recibían 300 colones/hora. El salario mínimo por hora en empacadoras vigente para esas fechas era de 321 colones/hora. Por lo tanto, las mujeres recibían menos del mínimo legal (Morales y Castro, 2008: 218).

Estas condiciones se ven acompañadas por otras de marcada precariedad, como el trabajo temporal y la extensión de los horarios de trabajo, y todas tienen el propósito de aumentar los márgenes de rentabilidad. Se trata de un comportamiento que la teoría agraria ha establecido como característica de las empresas en periodos en los cuales existe una sobre oferta de trabajo, situación que presenta la zona en estudio a causa de la abundancia de migrantes nicaragüenses. Esta necesidad se exacerba cuando los costos de producción de las empresas son altos. Tal comportamiento se generaliza para la mayor parte de la fuerza de trabajo contratada, sin importar el tamaño de las empresas.

"Las empresas agrícolas, mediante el deterioro de las remuneraciones a la fuerza de trabajo, han intentado compensar la elevación de los demás componentes del costo de producción, de mantener relativamente estable su nivel de rentabilidad y de conservar su vitalidad productiva. Con ello han favorecido el trabajo agrícola temporal en detrimento del permanente. Por su parte, las altas tasas de subempleo agrícola y rural han contribuido a la caída de los salarios agrícolas reales" (López, 1990: 372).

Dependiendo de la dinámica productiva en el sector agrícola, puede suceder que un nuevo producto de exportación o una empresa poderosa atraiga a trabajadores procedentes de distintos ámbitos del territorio nacional o a los mismos migrantes. En estos casos, las otras plantas procesadoras y fincas 
agrícolas pueden verse afectadas por la carencia de trabajadores, problema que hasta entonces no habían experimentado.

Si quieren retener a sus trabajadores no tendrán más remedio que aumentar las remuneraciones, pero ello perjudicará sus ganancias. Aunque estas situaciones se han presentado poco en la franja estudiada, el recurso principal para enfrentar esta situación ha consistido no en mejorar la innovación tecnológica y la productividad, sino en solicitar al gobierno que aumente las cuotas de migrantes temporales procedentes de Nicaragua y de otros países centroamericanos.

Se pone de manifiesto que las empresas y fincas agrícolas del país, para no limitar el análisis a la franja estudiada o al cantón de San Carlos, no tienen actividades cimentadas en el mejoramiento continuo deprocesos, ni aplican sistemáticamente herramientas tecnológicas para aumentar la productividad y la calidad de los productos, mejorando de paso su competitividad en los mercados externos, sino que sus plantas y fincas generan ganancias basadas en factores vinculados a la explotación de la fuerza de trabajo. Los análisis, entre otros, de Vargas (2002, 2007) y Láscaris (2004) hallan plena confirmación.

En el gráfico 3 se presenta la distribución de los trabajadores de las plantas procesadoras, según nacionalidad. Aunque la mayoría son costarricenses (57\%), hay una elevada proporción de nicaragüenses (43\%), lo que vuelve a confirmar la importancia laboral de esta mano de obra, especialmente en las labores agropecuarias y la agroindustria, tal y como señalan numerosos estudios y encuestas realizadas en Costa Rica (Estado de la Nación, 1997; Mora, 2007; Morales y Castro, 2008).

La presencia masiva de nicaragüenses en Costa Rica, sobre todo después de 1990, no puede entenderse sin el particular contexto socioeconómico del país vecino, y aunque el propósito de este trabajo no es presentar un análisis exhaustivo de las causas que promovieron la migración masiva de nicaragüenses a Costa Rica en las dos últimas décadas, se mencionarán algunas razones, partiendo del valioso y documentado estudio de los investigadores nicaragüenses Rocha y Terán (1998). Al analizar la situación de su país durante la segunda mitad de los años 1980 y primeros años de la década siguiente, estos autores concluyen que en el periodo indicado Nicaragua experimentó una pauperización acelerada en casi todos los sectores de la población; entre otras razones porque se aplicó a rajatabla un programa de ajuste en un país que venía saliendo de una durísima y traumática guerra, que no solo costó decenas de miles de muertos, heridos y mutilados, sino que además deterioró seriamente buena parte del aparato productivo y de la infraestructura física del país vecino. Diagnóstico coherente con el realizado por otros autores, como Torres (1993, 2007).

Rocha y Terán (1998) señalan que el ritmo de tal pauperización no fue gradual sino violento, y que mostraba nítidamente las señales de una economía deteriorada y en serio estado recesivo. Adicionalmente, uno de los rasgos sociales que presentaba Nicaragua durante los años en que se producen sucesivas oleadas de migrantes hacia Costa Rica consistía en lo que los autores denominan la "paulatina polarización de la pobreza" (p. 206), que consistía en la reducción de la brecha entre los hogares no pobres y los indigentes, que en 1985 era de $14.6 \%$ y en 1993 llegó al I I.3\%. Los autores prestan atención especial al estancamiento del mercado laboral en Nicaragua, que empujó a grandes contingentes de población hacia Estados Unidos y Costa Rica. Como corolario de sus análisis, Rocha y Terán concluyen:

"Es decir, se está ante un mercado laboral en que... las posibilidades de obtener una ocupación son muy limitadas. De ahí

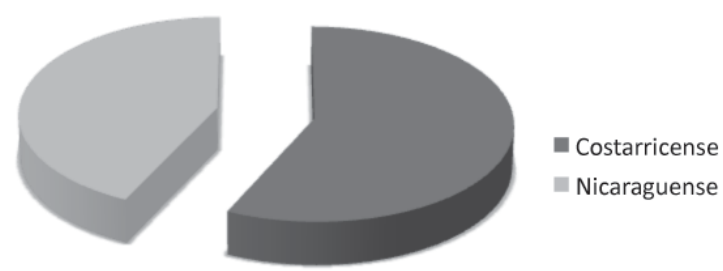

Gráfico 3. Distribución de los trabajadores en las empacadoras de yuca por nacionalidad (porcentaje). 
que el crecimiento de la PEA (población económicamente activa) se encuentre estancado y que dentro de ella sea el componente de desempleo abierto el que tiene una mayor significación... Las oportunidades de empleo se han reducido de manera significativa. El hecho de estar ante un mercado laboral sin dinamismo de generación de empleo y que ha incrementado dramáticamente la desocupación abierta, es el fenómeno más importante para entender cómo tal mercado está afectando el bienestar de los hogares nicaragüenses". (Rocha y Terán, 1998: 212 y 216. (Las cursivas son nuestras).

Cabría agregar un elemento más relacionado con la inmigración nicaragüense. Morales y Castro (2008) en un detallado estudio sobre la migración, el empleo y la pobreza, encontraron que la fuerza laboral nicaragüense mejor calificada y con escolaridad más alta se radica en el Gran Área Metropolitana de San José, mientras que la menos calificada y con menor escolaridad se establece en las zonas rurales y se emplea en la agricultura y la agroindustria (empacadoras, procesadoras, maquiladoras) de exportación.

Los resultados de la presente investigación confirman tales hallazgos, pues la escolaridad de la mano de obra nicaragüense en las empacadoras de la franja estudiada es muy baja. En efecto, el 25\% eran analfabetos, el 21.4\% sabían leer y escribir, aunque no habían cursado ningún año de estudios formales, mientras el 39.3\% contaban con educación primaria, fuera completa o incompleta. Solo el 14.3\% había cursado algún año de la educación secundaria.

Una de las variables sociales más importantes para conocer las condiciones de las familias, las circunstancias en que se reproduce el grupo familiary las expectativas futuras de ascenso social, o al menos de mantenimiento de las condiciones actuales, es el nivel de pobreza. Antes de presentar los resultados para los trabajadores nicaragüenses y costarricenses, se impone una explicación de la metodología utilizada para construir las categorías de la variable. Los datos y criterios utilizados corresponden a los aplicados por el Instituto Nacional de Estadísticas y Censos (INEP) en sus encuestas de hogares de propósitos múltiples, concretamente a los datos de julio del 2007, año en el cual se aplicaron las encuestas a los trabajadores de planta y eran, por lo tanto, los que debían usarse en el estudio.

Del INEC (2007) se tomaron los datos para construir las categorías de pobreza extrema (familias cuyos ingresos no les permiten cubrir el costo de la canasta básica alimentaria), de pobreza relativa (familias cuyos ingresos les permiten cubrir el costo de la canasta básica alimentaria, pero no el costo de otros servicios importantes, como vivienda, salud, educación o transporte) y de no pobres (familias cuyos ingresos les permiten cubrir los costos tanto de la canasta básica alimentaria como de los otros servicios). Para construir las categorías se utilizó el promedio de miembros promedio de una familia, que según INEC (2007) era de 3.9 en zona urbana y 4.2 en zona rural. Por ser la franja estudiada eminentemente rural, se tomó el último porcentaje.

Antes de presentar los resultados, es importante conocer las conclusiones a los cuales han llegado estudios precedentes que abordaron los niveles salariales en las actividades agropecuarias, especialmente en las no tradicionales de exportación, ya sea dentro o fuera de Costa Rica. Estos estudios coinciden en señalar que los salarios que se pagan en las actividades agrícolas son bajos, y ayudan apenas a cubrir los gastos de la canasta básica y otros servicios.

En otras palabras, son ingresos que se ubican por encima de la línea de pobreza, pero que no permiten acumular lo suficiente para acceder a niveles de vida cualitativamente diferentes a los que se tienen, con el inconveniente de que ciertos factores (como nacionalidad o género) llegan a incidir en menores prestaciones y, por lo tanto, en mayores posibilidades de situarse bajo el umbral de pobreza. Algunos autores también añaden como causa de los bajos salarios el hecho de tratarse de actividades muy básicas que, consecuentemente, requieren de una mano de obra poco calificada (Rojas y Román, 1993; Pérez y Pichardo, 1994; Estado de la Nación, 1996, 1997; Funkhouser y Pérez, 1998; Pérez, 1997, 2000; Tejo, 200 I; Fernández, 1994, 2004; Morales y Castro, 2008).

En el gráfico 4 se ofrece el nivel de pobreza para los trabajadores de origen nicaragüense. Los que se ubicaban dentro de la pobreza extrema constituyeron el 7\%; en la categoría de pobreza relativa (o no extrema o básica, como también la denomina el INEC) estaban el 39\%. Los no pobres eran el $43 \%$. Para establecer un marco comparativo 
de estos datos, aunque no sean de fecha reciente, se ofrecen los correspondientes al 200 I, referenciados por Morales y Castro (2008: I47), en cuyo estudio se comparaban, entre otros aspectos, los niveles de pobreza de los hogares costarricenses y nicaragüenses. En dicho trabajo se ponía de relieve que, entre las familias nicaragüenses, el $8.0 \%$ se ubicaba en la pobreza extrema; el 19.2\%, en la pobreza relativa, para un total de familias pobres del $27.1 \%$.

En comparación con los datos del estudio, hay porcentajes muy similares en la pobreza extrema, pero, en cambio, hay notables diferencias respecto a la pobreza relativa o básica: $19.2 \%$ reportado en el estudio de Morales y Castro (2007) sobre datos del INEC, y 39\% en la franja estudiada. Los niveles de pobreza para los nicaragüenses eran ligeramente mayores en las zonas rurales: así, la pobreza extrema se situaba en el $10.9 \%$ y la relativa en el $21.9 \%$, ascendiendo el porcentaje total de pobres rurales nicaragüenses al 32.9\%. Aún así, el porcentaje de pobreza relativa y total hallado entre las familias nicaragüenses que laboran en empacadoras de yuca de la franja estudiada continúa siendo mayor.

Los bajos niveles salariales, las pocas vinculaciones que las empresas exportadoras establecen con las economías locales o regionales y el que la mayor parte del valor quede fuera de estas o, incluso, fuera del país, provocan que "pese al crecimiento de la producción y de las ganancias de las empresas, los efectos sobre el desarrollo regional son débiles". (Faure y Samper, 2004: I5).
En el gráfico 5 se presentan los datos del nivel de pobreza para familias de los trabajadores costarricenses encuestados. El $90 \%$ de ellos se incluía en la categoría de no pobres, mientras el I0\% lo estaban dentro de la pobreza relativa. Ninguna se hallaba en situación de pobreza extrema. En este caso las cifras son mejores que para el conjunto de la región Huetar Norte o del país para el 2007, pues los porcentajes respectivos para estas últimas de no pobres fueron de $80.7 \%$ y $81.7 \%$ y los de pobreza relativa (o básico) fueron, también respectivamente, de $17.3 \%$ y de $14.0 \%$ (los de pobreza extrema alcanzaron porcentajes de $2.1 \%$ y $4.2 \%$ respectivamente) (INEC, 2007).

Hay que mencionar que para el 2007 la encuesta de empleo y propósitos múltiples que realiza el INEC reportó una importante baja en los niveles de pobreza, a nivel nacional y regional, luego de varios años que los niveles de pobreza estuvieron estancados. Esta situación generó polémica, pues según algunos estudiosos del tema no existían razones de peso para explicar esa baja, y, en cambio, la atribuían a un cambio "flexible" en la metodología para medirla. La baja, según su criterio, obedecía fundamentalmente a la aplicación de dicha metodología (cambios en el valor asignado a la canasta básica y a los servicios, y en la baja consecuente del nivel salarial para poder adquirirlos). Aquí no se entrará en la polémica, simplemente se le menciona.

En todo caso, y como principal recapitulación de lo indicado, para las familias costarricenses que obtenían sus ingresos trabajando en las empacadoras de la

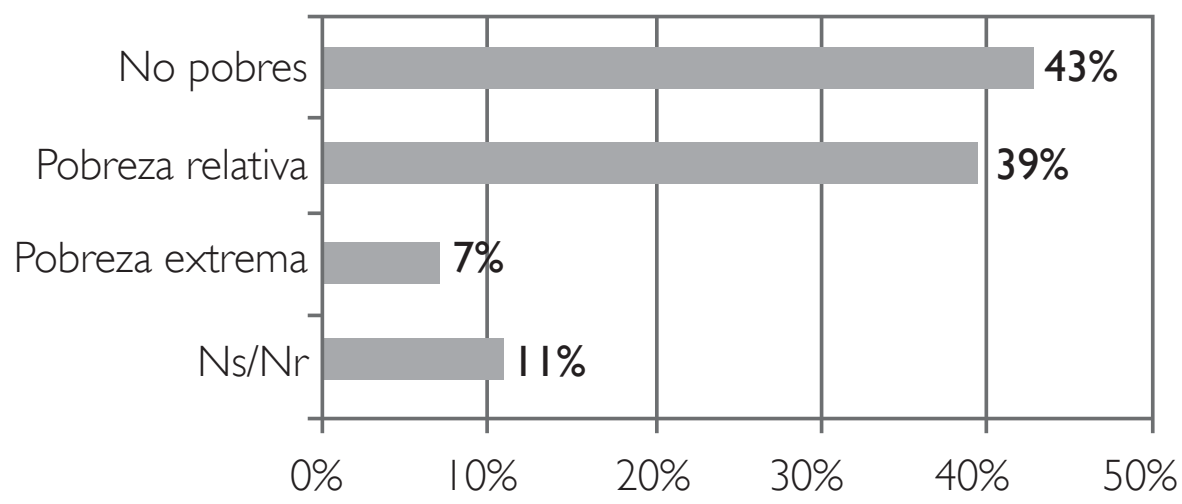

Gráfico 4. Nivel de pobreza de los trabajadores nicaragüenses de las empacadoras de yuca (porcentaje). 


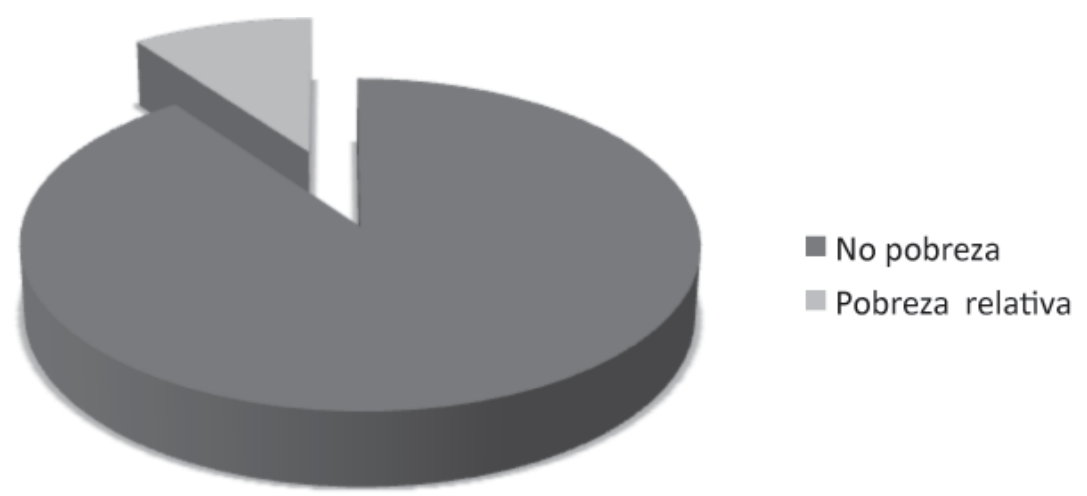

Gráfico 5. Nivel de pobreza de los trabajadores costarricenses de las empacadoras de yuca (porcentaje).

franja investigada, su situación, en cuanto a la pobreza se refiere, era mejor que para el conjunto de la región (Huetar Norte) y del país. La otra conclusión obvia es que a pesar de realizar los mismos trabajos que los nicaragüenses, sus ingresos son mayores, lo cual se refleja, como acaba de señalarse, en menores niveles de pobreza, sea relativa o extrema. En otras palabras, el factor nacionalidad pesa al momento de obtener beneficios que redunden en mejores condiciones de vida, pues no parecen existir otras razones que expliquen las diferencias salariales encontradas entre los trabajadores costarricenses y los nicaragüenses. En este y otros indicadores (como hacinamiento, seguro social), el trabajo de Morales y Castro (2008) llegaba a las mismas conclusiones.

Vale la pena detenerse en la tenencia de seguro social. Partiendo de los datos del censo de población del 2000, el estudio de Morales y Castro (2008: I 85) encontró cifras muy bajas de trabajadores nicaragüenses con seguro social: $58.4 \%$ para los hombres, 62.0\% para las mujeres y un total de 60.2\%. Para los nacionales costarricenses los porcentajes de aseguramiento fueron de $81.0 \%$ para los hombres, 85.7\% para las mujeres y un total de $83.4 \%$ de asegurados dentro de la población trabajadora. Los autores reafirman que la variable nacionalidad es la principal causa que explica tales diferencias. Sin embargo, en el presente estudio, a pesar de su provisionalidad y su carácter eminentemente exploratorio, no se hallaron diferencias en los porcentajes de tenencia de seguro social entre trabajadores costarricenses y nicaragüenses: en ambos casos el $90 \%$ de los trabajadores estaba asegurado. Será un estudio específico sobre el tema (y con una muestra representativa de trabajadores por nacionalidad) el que pueda llegar a conclusiones más satisfactorias.

Así, podrían formularse algunas hipótesis: un mayor control de la Caja Costarricense del Seguro Social, del Ministerio de Salud y del Ministerio de Trabajo; mayor conciencia de los empleadores o -y esto podría desprenderse de datos obtenidos en la investigación que exigirán, naturalmente, de ulterior prueba empírica- al hecho de que un alto porcentaje de los nicaragüenses tienen muchos años de residir en Costa Rica y, concretamente, en las comunidades de la franja estudiada. La mayoría expresó su deseo de quedarse en el país, tienen hijos nacidos en Costa Rica y los vínculos con su país de origen son cada vez más tenues. Es probable que sus empleadores los vean como "casi" costarricenses y por ello tienden menos a negarles el seguro social. No obstante, los salarios que se les pagan sí son menores que los recibidos por los trabajadores costarricenses, como se discutiera más arriba, y esto podría hacer creer más en el control que ejercen las autoridades competentes, en este caso las de la Caja, en lo referido al control sobre el pago y reporte del seguro social de los trabajadores.

\section{Conclusiones}

La mayor parte de la fuerza laboral de las empacadoras está formada por mujeres (63\%).

Los nicaragüenses constituyen un importante componente de la fuerza de trabajo de las empacadoras investigadas (43\%). 
La escolaridad hallada entre los trabajadores nicaragüenses encuestados es muy baja: el $25 \%$ eran analfabetos; el 21.4\% no sabían leer y escribir y el 39.3\% apenas contaba con educación primaria.

Los niveles de pobreza -tanto absoluta (7\%) como relativa (39\%) - son más altos entre los trabajadores nicaragüenses en relación a los costarricenses.

Se puede plantear como hipótesis de trabajo para futuros estudios sobre el tema que el factor nacionalidad influye negativamente en ciertas variables relevantes, como salarios más bajos y mayor incidencia de la pobreza.

En el estudio exploratorio no se encontraron diferencias por nacionalidad en cuanto a la tenencia de seguro social.

\section{Bibliografía}

Cordero, A. \& Mora, M. (1998). Costa Rica: El mercado de trabajo en el contexto del Ajuste. En: Funkhouser, Edward y Pérez, Juan (Ed.): Centroamérica en Reestructuración. Mercado laboral y pobreza en Centroamérica. San José, FLACSO, pp. 2 19-279.

Faure, G. \& Samper, M. (2004). Veinte años de apertura económica: el porvenir comprometido de la agricultura familiar en el norte de Costa Rica. En: Anuario de Estudios Centroamericanos (Costa Rica), Vol. 30(I y 2), 7-26.

Fernández, M. (2004). La agricultura costarricense ante la globalización. Las nuevas reglas del comercio internacional y su impacto en el agro. San José, Editorial de la Universidad de Costa Rica, 322 pp.

Fernández, M. (1994). La integración de la agricultura en la región centroamericana en el marco de los procesos de apertura. En: Masís, German y Sancho, Francisco (Comp.): La Agricultura de Exportación en Centroamérica: opciones de desarrollo en la década de los 90. San José, UNA, 1994, pp. 89- 152.

Funkhouser, E. \& Pérez, J. (1998). Ajuste Estructural, Mercado Laboral y Pobreza en Centroamérica. Una perspectiva regional. En: Funkhouser, Edward Pérez, Juan (Ed.): Centroamérica en Reestructuración. Mercado laboral y pobreza en Centroamérica. San José, FLACSO, pp. $281-333$.

Instituto Nacional de Estadística y Censos (INEC). (2007). Encuesta de Hogares de Propósitos Múltiples. Julio 2007. Principales Resultados. San José, CR, INEC, I 24 pp.
Láscaris, T. (2004). Innovación tecnológica y competitividad productiva en Costa Rica. Cartago, Editorial Tecnológica de Costa Rica, 156 pp.

López, L. (1990). Crisis, políticas del ajuste y agricultura. In: Núñez, Orlando. (Comp.): Lo agrario. Teoría y Métodos. San José, CR, CSUCA, pp. 353-395.

Mora, J. (2007). La vía cooperativa de desarrollo del agro. El caso de Copronaranjo R.L. Heredia, Costa Rica, Editorial Universidad Nacional, 262 pp.

Morales, A \& Castro, C. (2008). Migración, empleo y pobreza. San José, FLACSO, 274 p.

Pérez, L. (1997). Apertura Económica: ¿Ventajas o desventajas para la pequeña producción agropecuaria? En: Perspectivas Rurales (Costa Rica), No. I: 25-42.

Pérez, L. (2000). La pobreza rural analizada con enfoque de género. En: Perspectiva Rurales (Costa Rica), Año 4, No. I: 86- 120.

Pérez, L. \& Pichardo, A. (1995). Pobreza en el Istmo Centroamericano: Perspectiva de las mujeres. Tomo II. San José, Programa de las Naciones Unidas para el Desarrollo (PNUD), 155 pp.

Proyecto Estado de la Nación. Estado de la Nación en Desarrollo Humano Sostenible. Informes 1996 y 1997. San José, Proyecto Estado de la Nación.

Rivera, R. \& Román, I. ( 1990). Ajuste estructural y alternativas productivas para los pequeños productores. En: Reuben, William (Comp.): Los campesinos frente a la nueva década. Ajuste estructural y pequeña producción agropecuaria en Costa Rica. San José, Editorial Porvenir-CECADE, pp. $14 \mid-180$.

Rocha, J. \& Terán, J. ( 1998). Mercado laboral y pobreza en Nicaragua. En: Funkhouser, Edward y Pérez, Juan (Ed.): Centroamérica en Reestructuración. Mercado laboral y pobreza en Centroamérica. San José, FLACSO, pp. 175-2| 8.

Rodríguez, F. (2003 a). Caracterización de los Sistemas Productivos y de las Prácticas Culturales en la Subcuenca del río Peñas Blancas, cuenca del río San Carlos En: Pensamiento Actual (Costa Rica), Vol. 4 (5), 24-34.

Rodríguez, F. (2003 b). Formas de Laboreo, prácticas culturales y organización de la producción en la subcuenca del río Javillos. En:Tecnología en Marcha (Costa Rica), Vol. |6, ( I), 80-91.

Rodríguez, F. (2006). Formas de Laboreo, prácticas culturales y organización de la producción en la subcuenca del río Peñas Blancas, cuenca del río San Carlos. En: Rodríguez, Francisco y Chaves Adolfo (Comp.): Valoración del recurso hídrico de la cuenca del río San Carlos. Cartago, Taller de Publicaciones -del ITCR, pp. I06-I 44. 
Rodríguez, F; Chaves, A; Barrantes, U. \& Araya, F. (2004). Valoración de la condiciones del recurso hídrico de la cuenca del río San Carlos y sus efectos en la calidad de vida en la Región Huetar Norte, Costa Rica. Informe Final Proyecto de Investigación. Santa Clara, San Carlos, ITCR, Sede Regional San Carlos, Escuela de Ciencias y Letras.

Román, I. \& Rojas, M. ( 1993). Agricultura de exportación y pequeños propietarios en Costa Rica. San José, FLACSO (Cuadernos de Ciencias Sociales No 6I).

Romero, V.; Rodríguez, F.; jiménez, R. (2003). Eficacia de la legislación contra la violencia femenina y de las Oficinas de la Mujer, o instancias afines, en el ámbito laboral, público y privado de San Carlos. Informe Final Proyecto de Investigación. Instituto Tecnológico de Costa Rica, Sede Regional San Carlos, Escuela de Ciencias y Letras.

Stamm, A. (1996). ¿Una nueva dinámica para las zonas rurales? Relaciones intersectoriales y efectos territoriales del cambio en el modelo de desarrollo de Costa Rica. En: Nuhn, Helmut y Stamm, Andreas: Apertura comercial en Centroamérica: Nuevos retos para la agricultura. San José, Editorial DEl, pp. 23-48.

Tejo, P. (200 I). El modelo agrícola de América Latina en las últimas décadas. En: David, Beatriz (Comp.): Desarrollo
Rural en América Latina y el Caribe. Santa Fe de Bogotá, CEPAL-ALFAOMEGA, pp. 89-134.

Torres, E. (Ed.). (1993). Historia General de Centroamérica. Tomo VI. Historia Inmediata. Madrid, Sociedad Estatal Quinto Centenario-Facultad Latinoamericana de Ciencias Sociales, 253 pp.

Torres, E. (2007). La Piel de Centroamérica. (Una visión epidérmica de setenta y cinco años de su historia). San José, FLACSO, 286 pp.

Vargas, A. ( 1986). Sinopsis histórica del cantón de San Carlos. Ciudad Quesada, Municipalidad de San Carlos-Grupo Cultural Trapiche, 84 pp.

Vargas, L. (2002). Costa Rica. 1985-1997. Liberalización y ajuste estructural o la autodestrucción del Neoliberalismo. San José, EUNED, 374 pp.

Vargas, L. (2007). La estrategia de liberalización económica. (Periodo 1980-2000). San José, Serie Cuadernos de Historia de las Instituciones de Costa Rica, No. 9, Editorial de la Universidad de Costa Rica, 68 pp.

Weller, J. (1996). Efectos del ajuste estructural en el empleo y los ingresos agropecuarios, con énfasis en las exportaciones no tradicionales. Los casos de Costa Rica y Honduras. En: Nuhn, Helmut y Stamm, Andreas: Apertura comercial en Centroamérica: Nuevos retos para la agricultura. San José, Editorial DEl, pp. 195-224. 